\title{
Time is a rubberband: neuronal activity in monkey motor cortex in relation to time estimation
}

\author{
Louis Renoult, ${ }^{*}$ Sébastien Roux and Alexa Riehle \\ Mediterranean Institute of Cognitive Neuroscience (INCM), UMR 6193, CNRS \& Aix-Marseille Universities, Marseille, France
}

Keywords: pointing movements, reaction time, single-neuron recording, time estimation, variability

\begin{abstract}
Anticipation of predictable events is crucial for organizing motor performance. Using instructed delay tasks, it has been shown that even when delay duration is kept constant, reaction time fluctuates from trial to trial. As time estimation is at the core of anticipatory behavior, it is reasonable to speculate whether neuronal delay activity correlates with the subjective estimate of time. As a consequence of the scalar property of time estimation processes, the variability in time estimation increases continuously as time passes during the delay. This scalar property may then be reflected in the increasing variability in neuronal delay activity. We thus studied the influence of temporal prior information on neuronal delay activity in monkey motor cortex in two conceptually different tasks in which two equally probable delay durations were randomly presented. We hypothesized that if one considers the animal's subjective time as the time which elapses between the first (instruction) signal and movement onset, then, by suppressing this temporal variability, across-trial variability in neuronal discharge should decrease. We thus defined a new time scale in each trial such that, after rescaling, the time between the instruction signal and movement onset was identical in all trials. Each spike was then displaced in time accordingly. As expected, the variability in the timing of neuronal peak discharges no longer increased during the trial. This suggests a direct link between the temporal profile of spiking activity and time estimation. The timing of motor cortical activity reflected the 'elasticity' of the animal's subjective time.
\end{abstract}

\section{Introduction}

In motor tasks, mean reaction time is strongly reduced when temporal information is provided in advance (Riehle et al., 1997), but great trial-by-trial variability in movement initiation is often observed even though the delay duration remains constant over trials (Riehle \& Requin, 1993; Everling \& Muñoz, 2000). What is the reason for this variability? Factors such as the ever-changing level of arousal across trials and/or some uncontrollable (motor) noise may in part be responsible. Additionally, as the subject may estimate the delay duration, the variability in time estimation processes might have also a strong influence on behavior, and thus on neuronal activity. Interval timing systems are characterized by their relative imprecision (Gibbon et al., 1997); the standard deviation of time estimations grows proportionally with the mean of the duration to be estimated, as described by the scalar property of variance (Gibbon, 1977). Indeed, both time interval estimation and the persistent neuronal activity recorded in monkeys during imposed delay periods vary in time from trial to trial. Are these two parameters correlated? There is evidence that sensory and contextual signals may be accumulated, integrated and transformed to reach a threshold for action (Shadlen \& Newsome, 2001; Ditterich et al., 2003; Mazurek et al., 2003). The analysis of neuronal activity at the end of an instructed delay revealed a statistically significant trial-by-trial correlation between firing rate and

Correspondence: Dr A. Riehle, as above.

E-mail: alexa@incm.cnrs-mrs.fr

* Present address: Douglas Hospital Research Centre, McGill University, Frank B. Common Pavilion, 6875 LaSalle Blvd., Verdun, Montreal, Quebec H4H1R3, Canada.

Received 13 September 2005, revised 19 January 2006, accepted 16 March 2006 reaction time in a high proportion of motor cortical neurons: the higher the firing rate, the shorter the reaction time (Kubota \& Hamada, 1979; Riehle \& Requin, 1993; Riehle, 2005). Indeed, the trial-by-trial delay activity of individual motor cortical neurons reliably predicts movement performance, as expressed by reaction time (see also Hanes \& Schall, 1996; Dorris \& Muñoz, 1998, for the frontal eye field). Additionally, however, it is possible that not only is the amount of neuronal delay activity in terms of mean firing rate correlated with the temporal performance of the animal, but so too is the timing of spiking activity, e.g. its temporal profile. To investigate these possibilities, we studied the influence of temporal prior information on motor cortical activity in two conceptually different delayed pointing tasks. In both tasks, two equally probable delay durations were randomly presented to the monkey and multiple single neuron activity was recorded in primary motor cortex. We hypothesized that the animal would start time estimation at the beginning of the delay by resetting its internal time. Then, as a consequence of the scalar property of time estimation processes (Gibbon, 1977), the variability in time estimation should increase continuously as time passes during the delay. We furthermore hypothesized that this scalar property may be reflected in the timing of the spiking activity and in the increase of the across-trial variability in neuronal activity during the delay. We considered the animal's subjective time as the time which elapses between the first (preparatory) signal and movement onset and rescaled this time span accordingly to make it identical in all trials. We then displaced in each trial each spike in time using an appropriate algorithm. As expected, the across-trial variability in the timing of the peak locations of single trial firing rates no longer increased during the trial. Preliminary data have been presented in Renoult et al. (2004). 


\section{Materials and methods}

\section{Behavioral procedure}

Two male rhesus monkeys (monkey $\mathrm{Y}, 6 \mathrm{~kg}$, in task I, and monkey O, $5 \mathrm{~kg}$, in task II) were used for this study. They were cared for according to European Community's Council Directive for the Care and Use of Laboratory Animals (86/609/EEC).

In task I (see Riehle et al., 2000; Grammont \& Riehle, 2003), monkey $\mathrm{Y}$ sat in a primate chair in front of a vertical panel on which seven touch-sensitive, light-emitting diodes (LEDs) were mounted, one in the centre and six placed equidistantly on a circle around it. First, the center target was lit and the animal had to press it with the left hand to initiate the trial. Then, after a fixed delay of $500 \mathrm{~ms}$, the first signal (preparatory signal, PS) was presented by illuminating one of the targets in green. After another delay, during which the animal had to continue to press the center target, the color of the peripheral target turned to red. This served as the response signal (RS), requesting that the animal performs the movement as fast as possible in order to be rewarded by a drop of fruit juice. Two durations (600 and $1200 \mathrm{~ms}$ ) were presented at random with equal probability. Thus, the probability of the occurrence of the RS was 0.5 during the first $600 \mathrm{~ms}$, and 1 during the second half of the long delay. Each movement direction was presented at random with equal probability.

In task 2 (see Roux et al., 2003), monkey $\mathrm{O}$ was trained to execute movements in two opposite directions from a common center position. On a vertical panel, three touch-sensitive targets with LEDs (diameter, $3 \mathrm{~cm}$ ) were mounted in a horizontal line, $10 \mathrm{~cm}$ from each other. The animal self-initiated each trial by touching the center target with the active hand. After a fixed delay of $600 \mathrm{~ms}$, a PS was presented. Both peripheral targets were presented simultaneously, one in red and the other in green. The animal learned to attribute to each color one of two possible delay durations. If the (directionally non-informative) auditory RS occurred after a short delay $(600 \mathrm{~ms})$, the monkey had to select the red target, if it occurred after a long delay $(1200 \mathrm{~ms})$ the green one. Both the laterality of the colored targets and presentation of the two durations were varied at random with equal probability.

In both tasks, the probability of the RS occurring after a short delay was 0.5 . Once this duration had passed, its probability of occurring after a long delay was 1 . Furthermore, reaction time (RT, i.e. the time between RS and the release of the center target) and movement time (MT, i.e. the time between the release of the center target and touching the requested peripheral target) were limited to $600 \mathrm{~ms}$ each in monkey Y, and $300 \mathrm{~ms}$ in monkey $\mathrm{O}$.

\section{Electrophysiology}

After training, the animals were prepared for multiple single-neuron recordings. A cylindrical stainless steel recording chamber (inner diameter, $15 \mathrm{~mm}$ ) was implanted over the contralateral primary motor cortex (MI; see Fig. 1B for monkey Y) under aseptic conditions and general isoflurane anesthesia $(<2.5 \%$ in air). A stainless steel T-bar was cemented to the skull to fix the animal's head during recording sessions. In order to record extracellularly single-neuron activity from multiple sites, a multi-electrode, computer-controlled microdrive (monkey Y: Reitboeck system, Thomas Recording, Giessen, Germany; monkey O: MT-EPS, AlphaOmega, Nazareth, Israel) was used to insert several microelectrodes transdurally (monkey Y: up to seven quartz insulated platinum-tungsten electrodes, outer diameter $80 \mu \mathrm{m}$, impedance 2$5 \mathrm{M} \Omega$ at $1000 \mathrm{~Hz}$; monkey O: four epoxy-insulated tungsten electrodes, Frederick Haer, $0.5-1.2 \mathrm{M} \Omega$ at $1000 \mathrm{~Hz}$ ). From each electrode, electrical signals were amplified and band-pass filtered $(300 \mathrm{~Hz}$ to $10 \mathrm{kHz}$ ). Action potentials (spikes) of only one single neuron per elec- trode were then isolated by using a window discriminator (monkey $\mathrm{Y}$ ), or of 1-3 neurons per electrode by using online spike sorting with a template matching algorithm (MSD, AlphaOmega) in monkey O.

\section{Data analysis}

All analyses were performed in MATLAB (The MathWorks Inc., Natick, MA, USA). We concentrated our analysis on neuronal spiking activity recorded during long trials, which allowed us to distinguish between the expected occurrence of the RS at $600 \mathrm{~ms}$ [internal timing marker, expected signal (ES)] and then its actual occurrence at $1200 \mathrm{~ms}$ (external timing marker, RS).

The following selection criteria were applied to the recorded neurons: (i) at least 15 trials per trial type, (ii) a phasic modulation of activity, correlated with at least one of the three task events, i.e. after PS, around ES and RS; (iii) a minimal peak discharge rate of 20 spikes/s; and (iv) a reproducible pattern of discharge across trials. For each neuron, the discharge recorded during each movement direction was analysed separately, representing an independent data set.

In task I, at most six data sets could be selected for each neuron as a function of the six movement directions, whereas in task II, only two possible data sets were available (two movement directions).

To specify the link between the variability in neuronal activity and the variability in reaction time, we developed an algorithm for the temporal transformation of the spike data (see Figs 2 and 3). Precise spike times were determined on a trial-by-trial basis and a new time scale was defined in each trial such that the time between PS and movement onset was common for all trials (eliminating RT variability).

The new time scale was specifically defined for each data set. In order to obtain a new time scale that was comparable in size to the original one, we used as a reference time $t$ (ref) the duration of the long delay, i.e. the time between PS and RS, plus the median of RTs across all trials within this data set. In each trial, a timefactor was then determined by calculating

$$
\text { timefactor }(i)=t(i) / t(\text { ref })
$$

where $i$ is the actual trial number and $t(i)$ the duration of the PS - RS delay plus the RT in trial $i$. Thus, in each trial, time estimation is considered to be reset at PS corresponding to the time value of $t_{0}=0$. Each spike in trial $i$ was then displaced in time by multiplying its time of occurrence with the respective timefactor $(i)$.

We then estimated the firing rate in each single trial for both time scales using a convolution of spike occurrences with a triangular kernel of a standard width of $50 \mathrm{~ms}$ (Nawrot et al., 1999). The moments of peak discharge were determined in each trial during three time windows: after the PS, and around ES and RS (see Fig. 5A and B). Windows were determined individually for each dataset in respect to the peak occurrence measured in the poststimulus time histogram. Window sizes were $\pm 150 \mathrm{~ms}$ after PS and $\pm 200 \mathrm{~ms}$ around ES and RS, respectively. Peak latencies during both time scales were then measured and correlated with the behavioral response time in both the original and the new time scale.

\section{Results}

\section{Behavioral results}

The conditional probability for the RS to occur had direct consequences on reaction time. Mean RT (Fig. 1A, Table 1) in both tasks was significantly shorter after a long delay than after a short one. [Note that for simplicity we employ the term 'reaction time' here, although we are entirely aware of its questionable use in instructed delay tasks. 
A
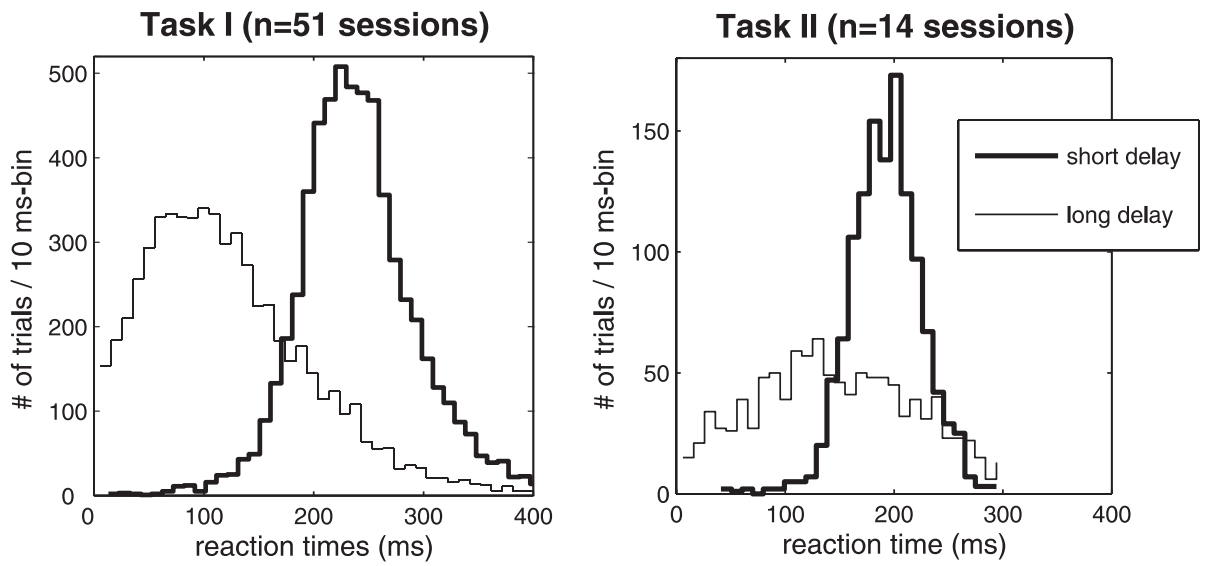

B

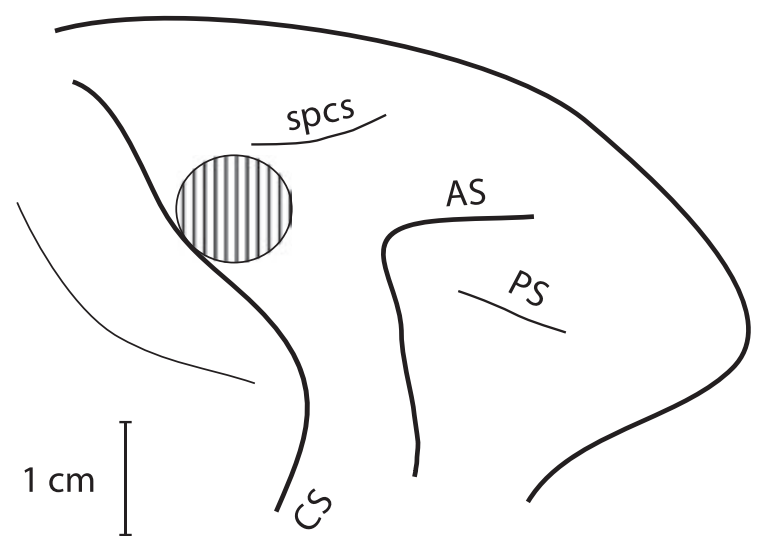

FIG. 1. (A) Behavioral results. Distributions of reaction times during all trials in all selected sessions for both monkeys. Only correct trials are shown in short (thick lines) and long (thin lines) delay trials. Left: task I, monkey Y, $n=51$ recording sessions, $n=5960$ short delay trials, and $n=5982$ long delay trials; right, task II, monkey $\mathrm{O}, n=14$ recording sessions, $n=1252$ short delay trials, and $n=1077$ long delay trials. Note that reaction time was limited to $600 \mathrm{~ms}$ in monkey Y and to $300 \mathrm{~ms}$ in monkey O. (B) Cortical surface representation of the right hemisphere of monkey Y. The hatched zone corresponds to the placement of the recording chamber. CS, central sulcus; AS, arcuate sulcus; PS, principal sulcus; spsc, superior precentral sulcus.

TABLE 1. Behavioral results in all selected recording sessions

\begin{tabular}{lllll}
\hline & & \multicolumn{2}{l}{ Reaction times $(\mathrm{ms})$} & \\
\cline { 3 - 5 } Task/monkey & $n$ & Short trials & Long trials & Difference \\
\hline Task I/Monkey Y & 51 & $238 \pm 59$ & $120 \pm 79$ & 118 \\
Task II/Monkey O & 14 & $188 \pm 15$ & $140 \pm 23$ & 48 \\
\hline
\end{tabular}

Data are presented as mean $\pm \mathrm{SD} . n=$ number of selected sessions.

It is evident that subjects do not necessarily react to the RS, but most often anticipate its occurrence.] When the RS did not occur $600 \mathrm{~ms}$ after the preparatory signal (PS, $P=0.5$ ), there was no longer any uncertainty regarding the moment of signal occurrence during the remaining $600 \mathrm{~ms}$ of the long delay $(P=1)$ and, thus, the animals initiated the requested movement earlier. This effect was stronger in monkey Y (difference in RT between long and short trails, $118 \mathrm{~ms}$ ) than in monkey $\mathrm{O}$ (difference, $48 \mathrm{~ms}$ ).

In parallel with the mean reduction in RT for long delays, variability in RT (in terms of standard deviation) was larger after long delays than after short delays (Fig. 1A). Moreover, the distribution of reaction times showed numerous anticipations in long trials with the movement initiated only a few milliseconds after RS, revealing an appropriate estimation of the delay duration. This was even true in task I where no explicit estimation was required (see also note above).

\section{Electrophysiological results}

\section{Discharge patterns}

We recorded the activity of 493 task-related neurons in primary motor cortex in two monkeys (recording site of monkey Y, hatched zone in Fig. 1B). The selection criteria were applied to the neuronal discharge during each movement direction (see Materials and methods). If the criteria were fulfilled, the neuronal activity recorded during this movement direction was used as an independent data set. Thus, theoretically the activity of one single neuron could be split into six different data sets in task I, and two different data sets in task II. Consequently, among 359 task-related neurons recorded in monkey Y in task I, 255 data sets were selected for further analysis, whereas in task II (monkey O) only 32 data sets were selected among 134 taskrelated neurons. Thus, among all task-related neurons $(n=493), 287$ data sets were selected for further analysis (see Table 2 ) and named for simplicity in the following 'neurons'. 

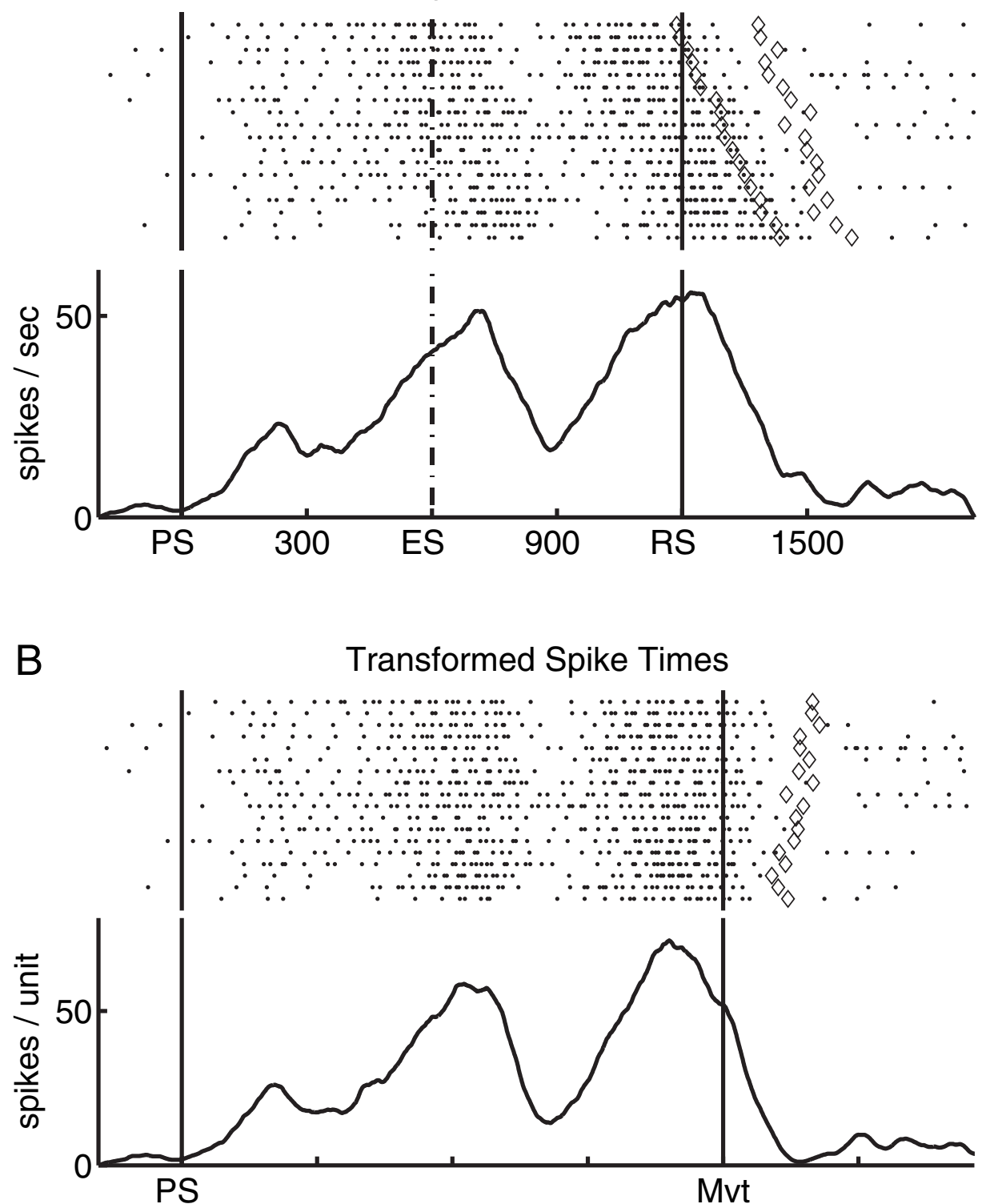

yo045-5-1

FIG. 2. Activity of a neuron recorded in monkey Y during task I in a long delay trial, before (A) and after (B) time transformation. Data are presented both as raster display and PSTH. Trials were arranged off-line according to increasing reaction time, from top to bottom. Note that because in long trials the probability of signal occurrence was 1 at the end of the delay period, the animals produced many anticipations and, thus, 'reaction' time may be very short by almost synchronizing the response with the occurrence of the response signal. In A, neuronal spike data are presented in the original time scale (time in ms). The data are aligned to signal occurrence. PS, preparatory signal; ES, expected (response) signal; RS, response signal. The first row of diamonds corresponds to movement onset, the second one to movement end. In B, the same data are presented after the trial-by-trial transformation of the time scale, the time scale being normalized between PS ( $\left.t_{0}\right)$ and movement onset. Here, the diamonds correspond to movement end.

TABLE 2. Numbers of task-related neurons

\begin{tabular}{llcrrr}
\hline Task/monkey & Total task- related neurons & Selected data sets & Analysed during PS & Analysed during ES & Analysed during RS \\
\hline Task I/Monkey Y & 359 & 255 & 96 & 211 & 198 \\
Task II/Monkey O & 134 & 32 & 8 & 24 & 32 \\
Task I \& II/2 monkeys & 493 & 287 & 104 & 235 & 230 \\
\hline
\end{tabular}

In Fig. 2A (task I) and Fig. 3A (task II) typical examples of the discharge pattern of selected neurons recorded during long trials are shown. In both tasks, the neurons, which were almost silent before the occurrence of the PS, increased their discharge after PS and then peaked a first time around the moment when the first RS was expected (ES), and finally before movement onset. 

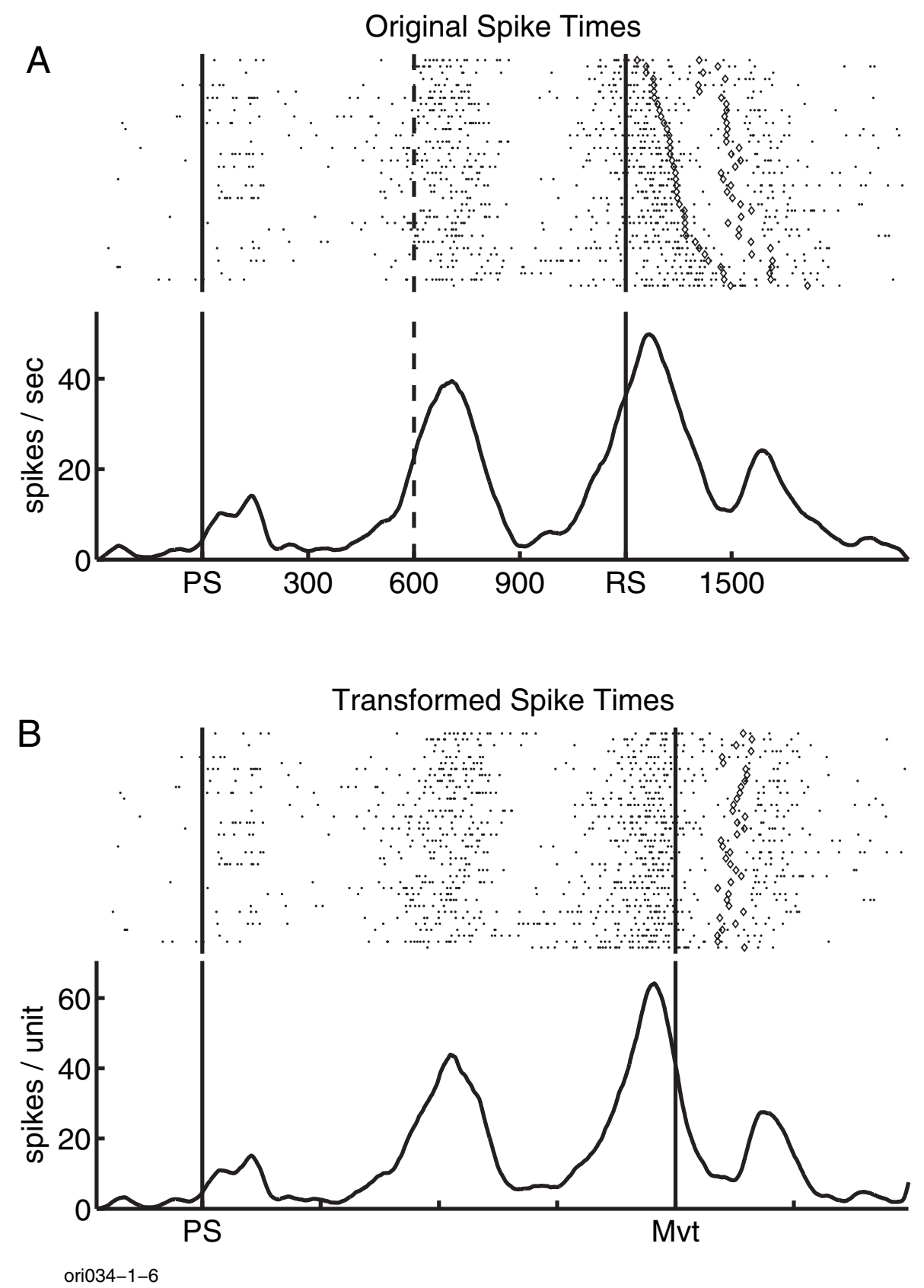

FIG. 3. Activity of a neuron recorded in monkey O during task II in a long delay trial, before (A) and after (B) time tranformation. For further details, see Fig. 2.

We then defined a new time scale in each trial such that after rescaling, the time between PS and movement onset was identical across trials (see Materials and methods). Each spike was then displaced in time accordingly. Indeed, after transformation of the time scale, the discharge pattern of the neurons became highly stationary across trials, as can be seen in the raster displays showing the spiking activity of the two individual neurons in Figs $2 \mathrm{~B}$ and 3B.

Figure 4 shows the averaged activity of the whole population of selected neurons $(n=287)$ for each of the two monkeys in both the original (Fig. 4A) and the transformed time scale (Fig. 4B). It can clearly be seen that, at the population level, mean activity increased as a function of the conditional probability during the trial in the same way for both monkeys. The main difference between the two monkeys was that the timing of the peak occurrences around ES was slightly shifted. This shift, however, corresponded to the difference in the mean RTs in short trials. RTs are indicated by the short vertical lines after ES in Fig. 4A and correspond to the mean values of movement onset if a short trial had been performed.

\section{Correlation between neuronal discharge and reaction time}

In order to examine the relationship between neuronal activity and RT, we analysed, as a first step, the correlation between the number of spikes during the delay period and RT. This correlation was expected to be negative, i.e. the higher the neuronal activity the shorter the reaction time. In $27 \%$ of the neurons $(78 / 287)$, the correlation was statistically significant $(P<0.05)$ in the original time scale. Interestingly, but not surprisingly, this negative correlation dropped to $4.5 \%(13 / 287)$ in the transformed data. Note that in the transformed data, the number of spikes was counted during the delay between PS and movement onset. 
A

Original Spike Times

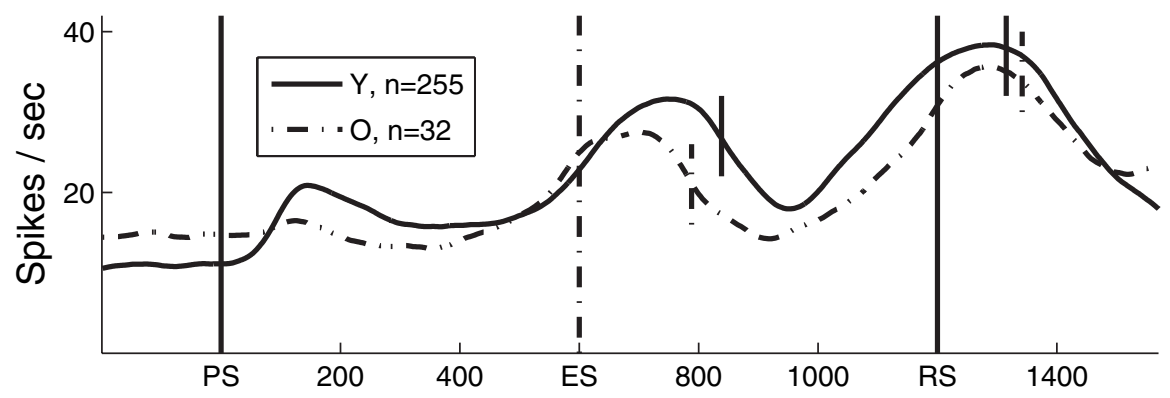

B

Transformed Spike Times

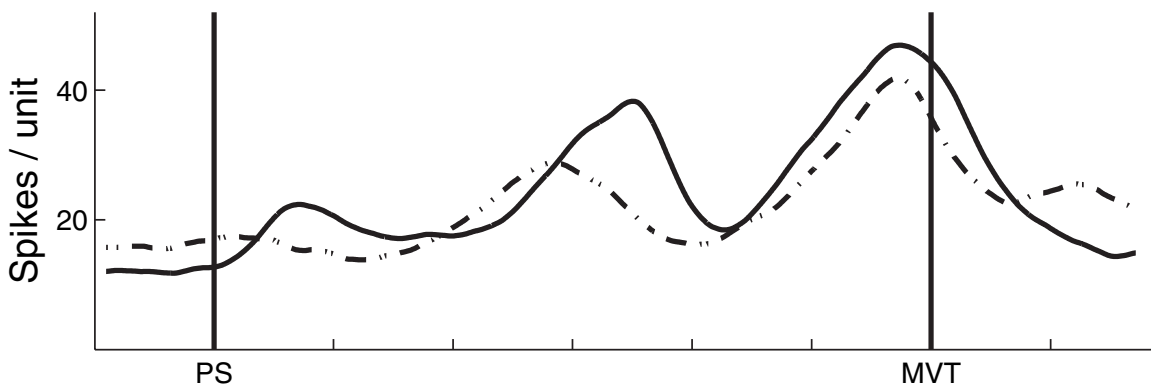

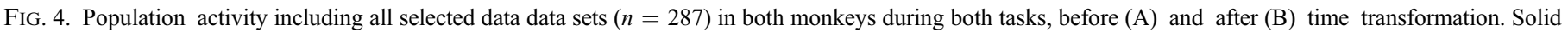

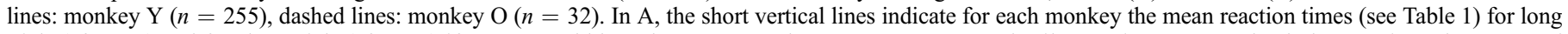

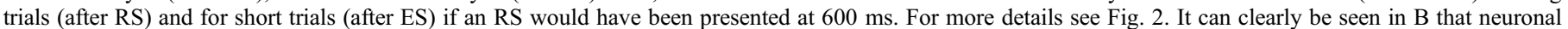
activity peaks shortly before movement onset in both monkeys at about the same time.

We then estimated single trial firing rates by convoluting trial-bytrial spike data using a triangular kernel with $50 \mathrm{~ms}$ standard width (Nawrot et al., 1999; cf Material and methods and Fig. 5A and B). Using these single trial firing rate estimations, we calculated the correlation between the peak firing rate during the last window around RS (see below). Here, the correlation was significant in only $2.6 \%$ $(6 / 230)$ of the neurons in the original time scale, and in $5.2 \%$ $(12 / 230)$ in the transformed time scale.

\section{Correlation between peak latencies and reaction time}

For quantifying the variability in the temporal occurrence of neuronal discharge across trials, we determined for each data set three time windows around PS, ES and RS (see Fig. 5A and B) containing the moments of activity peaks. We then measured the latencies of the peaks for each time window and investigated the relationship between the temporal variability in neuronal discharge and reaction time by computing correlations between peak latencies and RT (see Fig. 5C for an individual example). As shown in Table 3, most neurons $(86.1 \%$, $198 / 230)$ showed significant $(P<0.05)$ positive correlations between peak latencies and RT in the original time scale around RS. This correlation indicates that the later the neuron discharged within the selected time window, the longer the reaction time. The number of neurons exhibiting a significant correlation was smaller around ES $(51.1 \%, 120 / 235)$, and it decreased even more in relation to PS $(12.5 \%, 13 / 104)$. In the modified time scale, we expected these correlations to be drastically diminished. Indeed, significant correlations were extremely rare in this case (Table 3).

\section{Variability in peak latencies}

We then studied the across-trial variability of the timing of the peak occurrences and calculated the standard deviations of the peak latencies in each time window. In the original time scale in both tasks, the across-trial variability of peak latencies increased continuously as time elapsed during the trial (see Fig. 5D for a single neuron). This was confirmed for the whole population of neurons (Fig. 6A). In both tasks, standard deviation of peak latencies were smallest at the beginning of the trial (PS) when the animal was supposed to start time estimation, increased significantly during the moment when the first RS was expected (ES) $(t=5.12$, d.f. $=337, P<0.0001)$ and increased further significantly toward RS $(t=6.74$, d.f. $=463$, $P<0.0001)$. However, using the modified time scale, the variability in the single neuron data (see Fig. 5D) remained small and constant during the time course of the trial. For the whole population of neurons (Fig. 6A), the small difference in standard deviation between PS and ES was much less significant $(t=2.66$, d.f. $=337, P<0.01)$ and between ES and RS the variability in peak latencies even decreased slightly $(t=1.95$, d.f. $=463, P<0.05)$.

The difference in standard deviations of peak latencies before and after the application of the time transformation algorithm (see Fig. 6B for the population data) was not significant around PS $(t=0.77$, d.f. $=206, P=0.44$, n.s.), but was highly significant around ES $(t=4.02$, d.f. $=468, P<0.0001)$ and even more so around RS ( $t=11.1$, d.f. $=458, P<0.0001)$. The modified time scale thus resulted in a realignment of discharge peaks during behaviorally relevant trial epochs.

\section{Correlation between mean latency and correlation coefficient}

As mentioned above, we expected the correlation between peak latencies and reaction time to drop to zero in the transformed data. Interestingly, however, in some cases this correlation even produced negative values, which could be statistically significant (see Table 3). The question then arose as to why the correlations became negative. It 

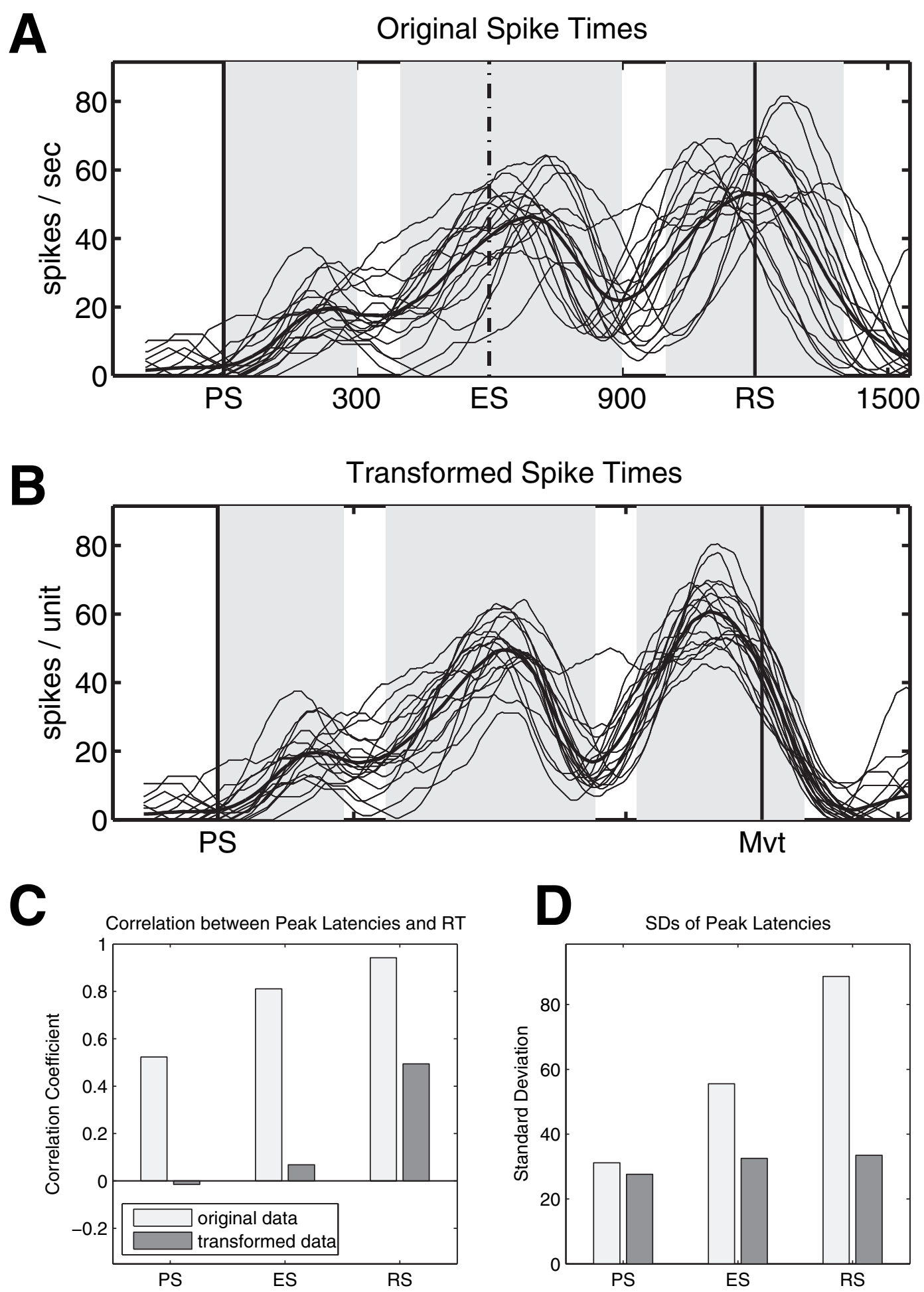

yo045-5-1

FIG. 5. Single-trial rate estimations for the same neuron as Fig. 2 (task I). Each thin curve represents the estimated discharge rate in a single trial. The thick line is the averaged estimated discharge rate. The grey areas around the task events correspond to the selected time windows for measuring peak latencies. (A) Trial-bytrial discharge in the original spike time. (B) The same data after transformation of the spike times. (C) Correlations between peak latency and reaction time (RT) measured with respect to the selected time windows (grey areas in A and B) after the preparatory signal (PS), around the expected signal (ES) and around the response signal (RS). Latencies were determined in the original data (light grey) and transformed data (dark grey). (D) Standard deviations (SDs) of peak latencies determined in the same selected windows, in both the original (light grey) and the transformed (dark grey) data. 
TABLE 3. Numbers and percentages of statistically significant correlations between peak latency and reaction time in tasks I and II*

\begin{tabular}{|c|c|c|c|c|c|c|}
\hline & \multicolumn{3}{|c|}{ Negative correlations } & \multicolumn{3}{|c|}{ Positive correlations } \\
\hline & PS $(n=104)$ & $\mathrm{ES}(n=235)$ & $\mathrm{RS}(n=230)$ & PS $(n=104)$ & $\mathrm{ES}(n=235)$ & $\mathrm{RS} \quad(n=230)$ \\
\hline \multicolumn{7}{|l|}{ Original data } \\
\hline Numbers & 1 & 0 & 0 & 13 & 120 & 198 \\
\hline Percentages & 1 & 0 & 0 & 12.5 & 51.1 & 86.1 \\
\hline \multicolumn{7}{|c|}{ Transformed data } \\
\hline Numbers & 3 & 8 & 19 & 7 & 10 & 9 \\
\hline Percentages & 2.9 & 3.4 & 8.3 & 6.7 & 4.3 & 3.9 \\
\hline
\end{tabular}

$* P<0.05$ (Monkey Y and Monkey $\mathrm{O}, n=287$ ).
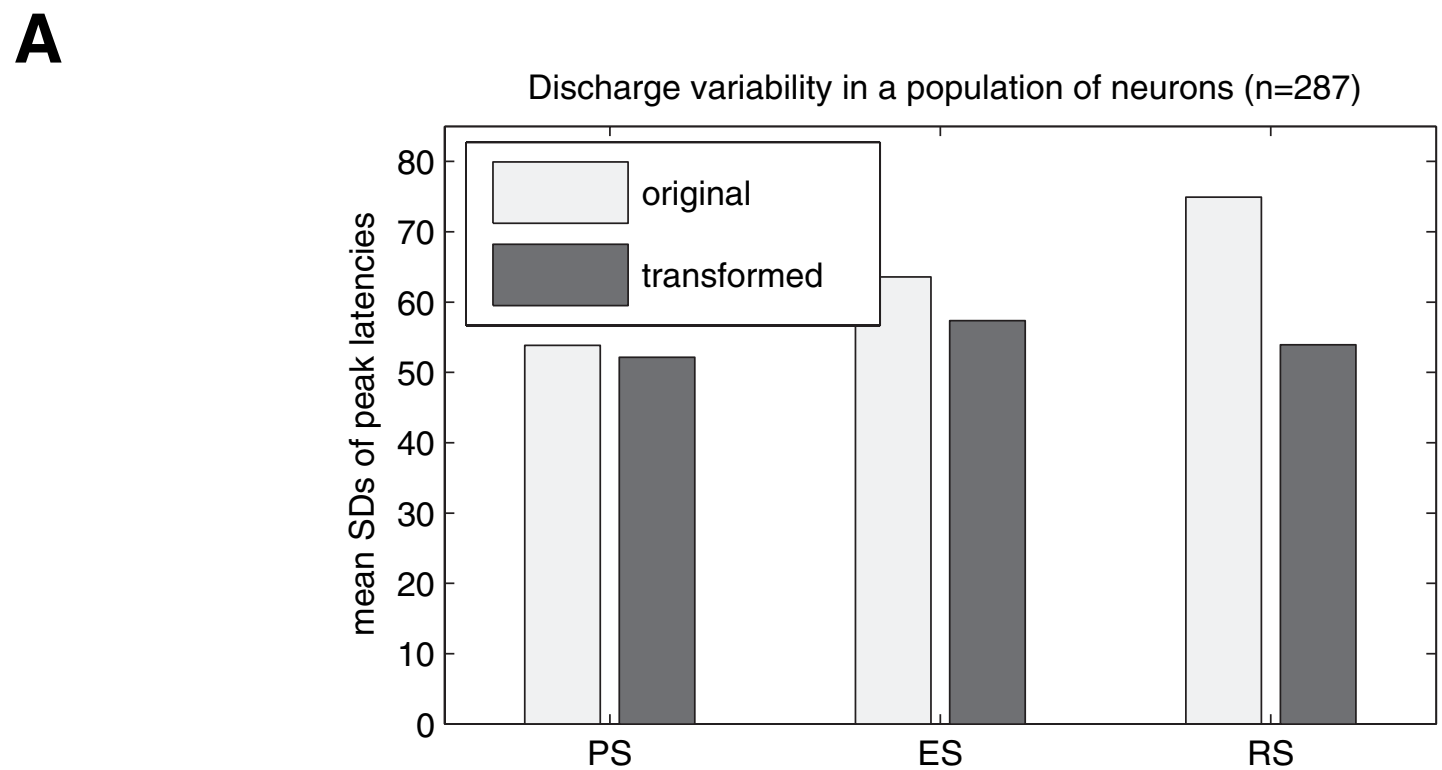

B

$\mathrm{t}=0.77$, ns

$t=4, p<0.0001$

$t=11.1, p<<0.0001$
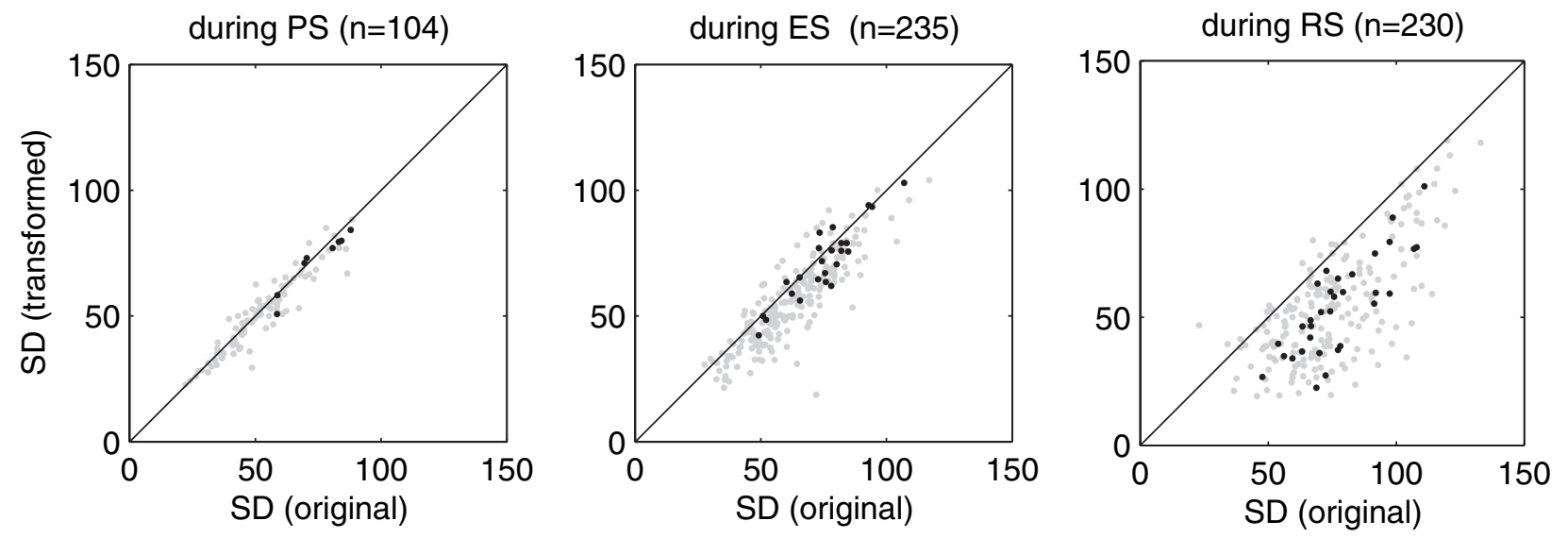

FIG. 6. (A) Mean standard deviations (SDs) of peak latencies of the whole population of neuronal data $(n=287)$ recorded in both tasks. Light grey, original time scale; dark grey, transformed time scale (see Fig. 5C for a single neuron). PS, preparatory signal; ES, expected signal; RS, response signal. Below each data set, the statistical significance for the difference between original and transformed time scale is indicated (see text). (B) Correlation between standard deviations (SDs) obtained in the original time scale [SD (original): $x$-axis] and that of the transformed time scale [SD (transformed): $y$-axis] in both tasks around PS (left), ES (middle) and RS (right). Light grey dots: monkey Y; black dots: monkey O. It can clearly be seen that the data from the two monkeys (and thus two different tasks) are identical.

appeared that the later a neuron discharged with respect to movement onset the more negative was the correlation. To quantify this relationship, we performed an analysis for the entire population in which we first calculated the correlation between peak latency around movement onset in the transformed data and reaction time (y-axis in Fig. 7). The resulting correlation coefficients, whether significant or 


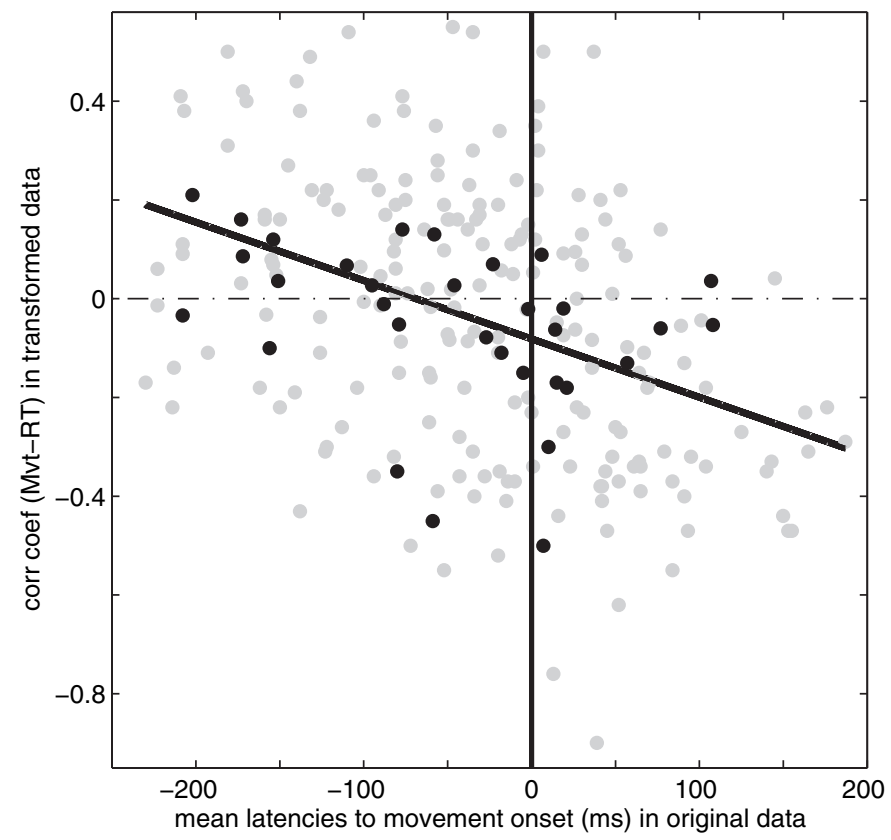

FIG. 7. Correlation between the mean latency in respect to movement onset in the original data ( $x$-axis) and the correlation coefficient of the correlation between peak latency in respect to movement onset and reaction time in the transformed data ( $y$-axis). It can be seen that there is a clear relationship between these two measures $(r=-0.401$, d.f. $=228, P<0.0001)$. Light grey dots: monkey Y; black dots: monkey O. The later the neuron discharged with respect to movement onset the more negative was the correlation. The zero crossing of the linear regression line is at $-69 \mathrm{~ms}$. See text for further discussion.

not, were then correlated with the mean peak latencies with respect to movement onset in the original data ( $x$-axis). Indeed, there was a clear relationship between these two measures $(r=-0.401$, d.f. $=228$, $P<0.0001)$. Logically, the timing of the peak activity of a typical movement-related neuron should be uncorrelated with reaction time in the transformed data $(r=0)$ because its trial-by-trial activity is perfectly aligned to movement onset. In our population, the mean peak latency of such perfectly aligned movement-related neurons appeared to be $-69 \mathrm{~ms}$, corresponding to the zero-crossing of the regression line.

\section{Discussion}

\section{Time estimation is at the core of anticipatory behavior}

In order to study anticipatory processes, tasks in which a delay is imposed between two signals are frequently used. Persistent neuronal activity recorded in monkeys during such delays has mostly been interpreted as linked to memory processes (Goldman-Rakic, 1996; Fuster, 2001; Wang, 2001; Brunel, 2003), attention (Simon et al., 2002), motor preparatory processes (reviewed by Evarts et al., 1984; Riehle, 2005) or decision processes (Leon \& Shadlen, 2003), partly as a function of the cortical area under study. However, only a few studies using single neuron recordings have postulated a link between delay activity and timing processes. In a pioneering study, Niki \& Watanabe (1979) interpreted delay activity as possibly linked to time estimation, and showed anticipatory changes in delay activity in prefrontal cortex. The authors referred to similar anticipatory patterns in motor cortex during reaction time tasks (Schmidt et al., 1974; Tanji \& Evarts, 1976) that had been interpreted differently. Only later was delay activity recorded in premotor cortex interpreted as being timing- related (Mauritz \& Wise, 1986; Vaadia et al., 1988). Here, delay activity was considered to be related to the anticipation of predictable events. Neural correlates of timing processes are now repeatedly described in different cortical areas (Lucchetti \& Bon, 2001; Leon \& Shadlen, 2003; Roux et al., 2003; Janssen \& Shadlen, 2005). Recently, Brody et al. (2003) found that the activity of a majority of prefrontal neurons varied systematically during the delay period in a time-related manner. They suggested that 'this time-dependent activity may encode time itself and may be an intrinsic part of active memory maintenance mechanisms' (p. 1196). Using a visuomotor temporal discrimination task, Leon \& Shadlen (2003) found neurons in posterior parietal cortex that reflected the time-dependent probability for a particular stimulus in their response field becoming the target of a saccade. In this context, time estimation processes and decisional processes were discussed as being tightly linked (see also Gold \& Shadlen, 2000; Mazurek et al., 2003).

Although time estimation was not mandatory in task I, the behavioral data strongly suggest that the duration of the delays was correctly estimated in both tasks. Reaction time distributions during long trials revealed numerous anticipations. This is incompatible with a strategy according to which the animal would have simply awaited the response signal to initiate its movement in task I. Indeed, when the signal did not occur after the short delay, the probability of its occurrence increased to 1 and the animal initiated the movement earlier, suggesting an appropriate estimation of the delay duration as a function of conditional probability (see Requin et al., 1991; Riehle et al., 1997). Accordingly, mean reaction times were much shorter after a long delay than after a short one in both tasks (see also Grammont \& Riehle, 2003; Roux et al., 2003). Moreover, in both tasks, variability in reaction time (in terms of standard deviation) was much larger after long delays than after short ones (see Fig. 1A). This can, at least partly, be explained by the scalar property of time estimation processes (Gibbon, 1977), suggesting that the distribution of reaction times should be larger after long delays than after short ones.

\section{Correlation between the timing of spiking activity and movement performance}

The across-trial variability in the latencies of the discharge peaks increased with time in the original time scale in both tasks. One might then wonder if this less and less precise alignment of peak occurrences across trials, from the preparatory signal to the response signal, is linked to the variable time of movement initiation, which in turn may be, at least partly, an effect of the variability in the animal's time estimations. Indeed, the temporal variability in peak occurrences decreased strongly in the new time scale, in which the variability of the moment of movement initiation was suppressed (transformed data). Moreover, most neurons showed significant correlations between peak latencies and reaction time, not only around the response signal, but also around the expected signal, a purely internal cognitive event. The temporal profile of neuronal discharge during the entire delay period was therefore highly correlated with the temporal organization of movement performance, and this was long before movement initiation. Furthermore, the fact that the increase in the variability of peak latencies during the time course of the trial concurred with an increase in the proportion of significant correlations between peak latencies and reaction time suggests an implication of precisely timed spiking activity in time estimation processes which are modified trial-by-trial as a function of the animal's subjective time scale. This observation constitutes an interesting way to predict the 
scalar property of time estimation based on the study of the temporal variability of neuronal activity. Note that single neurons in primary motor cortex synchronize their activity around the expected occurrence of an event (Riehle et al., 1997). This phasic emergence of synchrony may be a result of the event-related peak discharges, which may be used to indicate the end of an estimated delay.

There is evidence for a strong correlation between the mean firing rate during an instructed delay, i.e. the number of spikes, and the subsequent movement performance, expressed by reaction time. This has been described for various cortical areas and in relation to both arm movements (Kubota \& Hamada, 1979; Lecas et al., 1986; Riehle \& Requin, 1993; the present study) and saccadic eye movements (Hanes \& Schall, 1996; Dorris \& Muñoz, 1998). However, the proportion of neurons whose mean firing rate was significantly correlated with reaction time was never as high as the proportion of neurons whose temporal profile was significantly correlated with RT. Finally, the lowest proportion was that showing a correlation between the peak firing rate and RT. As a result, the timing of spiking activity seems thus to provide the best prediction for performance speed compared with mean firing rate and peak firing rate.

\section{Timing information and motor representation}

The displacement of spikes in time by using our transformation algorithm did not totally suppress the across-trial variability. The hypothesis of a continuous process of time estimation observable in the discharge patterns of motor cortical neurons from $t_{0}$, i.e. the preparatory signal, to movement initiation is only the simplest hypothesis. A strong argument in favor of this hypothesis is the fact that neurons whose peak latencies in respect to the response signal were not correlated with reaction time in the transformed data, an activity pattern that may be defined as purely movement-related, peaked at about $70 \mathrm{~ms}$ before movement onset (see Fig. 7). Indeed, this latency corresponds to the mean peak discharge latency of wellcontrolled motor cortical output neurons (see Evarts, 1974; Thach, 1978). However, we cannot exclude the possibility that the animal may change its representation of time during the trial or between trials by accelerating or decelerating at any time.

How time coding in motor cortical activity interacts with spatial coding, i.e. coding of directional parameters, will be an important question for future research (see also Mauk \& Buonomano, 2004). Classically, a large percentage of motor cortical neurons are characterized by their directional tuning, including a preferred direction (Georgopoulos, 1991). Here, we analysed neuronal activity recorded during each movement direction separately as an independent data set. As a minimal peak discharge rate of 20 spikes/s was one of our selection criteria, the relative increase in the discharge rate for each neuron in its preferred direction may have partly influenced our selection of data. Our results are nonetheless compatible with the hypothesis that timing parameters in motor cortical activity might be relatively independent of directional parameters. Vaadia et al. (1988) compared the delay activity recorded in premotor cortex in conditions with and without prior information about movement direction and studied its time dependency. Most of their neurons showed anticipatory timed activity independently of directional information. It seems that as the uncertainty concerning movement direction decreases, firing rate increases in monkey motor cortex (Bastian et al., 2003) and spatial and temporal representations in neuronal activity become more salient (Roux et al., 2003). Timing information and spatial parameters could thus be embedded within a global context-dependent motor representation (see also Roux et al., 2003).
We showed in two delayed motor tasks that the across-trial variability in the moment of movement initiation (i.e. reaction time) was reflected in the timing of neuronal delay activity recorded in primary motor cortex. Moreover, we described the same type of neuron both in a time estimation task (task II) and in a classical preparation paradigm (task I). Indeed, in typical instructed delay tasks, although time estimation may not be necessarily required, the considerable amount of training received by the animals to achieve a high performance level suggests the formation of strong memory traces of the time structure of the delays. This can be generalized to the potential influence of long-term training in animal studies on time estimation processes. It is possible that the neuronal representation of time is made locally more salient by strong memory traces of the delays between stimulus and response. Indeed, the implicit link between timing aptitudes of animals and general cognitive faculties such as memory, attention and decision processes (Mangels et al., 1998) is well described by the main models of interval timing (Ivry, 1996; Gibbon et al., 1997; Staddon \& Higa, 1999). The relative importance of each of these faculties in conjunction with the delay duration itself, and the role of potential central clocks in the brain still need further investigation.

\section{Acknowledgements}

We thank Bjørg Kilavik, Markus Diesmann, Sonja Grün, Martin Nawrot and Stefan Rotter for help in data analysis and valuable discussions. The research was supported in part by the CNRS and the French Government (ACI Cognitique 'Variability \& Invariants').

\section{Abbreviations}

ES, expected (response) signal; MI, primary motor cortex; MT, movement time; PS, preparatory signal; RS, response signal; RT, reaction time.

\section{References}

Bastian, A., Schöner, G. \& Riehle, A. (2003) Preshaping and continuous evolution of motor cortical representations during movement preparation. Eur. J. Neurosci., 18, 2047-2058.

Brody, C.D., Hernandez, A., Zainos, A. \& Romo, R. (2003) Timing and neural encoding of somatosensory parametric working memory in macaque prefrontal cortex. Cereb. Cortex, 13, 1196-1207.

Brunel, N. (2003) Dynamics and plasticity of stimulus-selective persistant activity in cortical network models. Cereb. Cortex, 13, 1151-1161.

Ditterich, J., Mazurek, M.E. \& Shadlen, M.N. (2003) Microstimulation of visual cortex affects speed of perceptual decisions. Nat. Neurosci., 6, 891-898.

Dorris, M.C. \& Muñoz, D.P. (1998) Saccadic probability influences motor preparation signals and time to saccadic initiation. J. Neurosci., 17, 70157026.

Evarts, E.V. (1974) Precentral and postcentral cortical activity in association with visually triggered movement. J. Neurophysiol., 37, 373-381.

Evarts, E.V., Shinoda, Y. \& Wise, S.P. (1984) Neurophysiological Approaches to Higher Brain Functions. John Wiley \& Sons, New York.

Everling, S. \& Muñoz, D.P. (2000) Neuronal correlates for preparatory set associated with pro-saccades and anti-saccades in the primate frontal eye field. J. Neurosci., 20, 387-400.

Fuster, J.M. (2001) The prefrontal cortex - an update: time is of the essence. Neuron, 30, 319-333.

Georgopoulos, A.P. (1991) Higher order motor control. Annu. Rev. Neurosci., 14, 361-377.

Gibbon, J. (1977) Scalar expectancy theory and Weber's law in animal timing. Psych. Rev., 84, 279-325.

Gibbon, J., Malapani, C., Dale, C.L. \& Gallistel, C.R. (1997) Towards a neurobiology of temporal cognition, advances and challenges. Curr. Opin. Neurobiol., 7, 170-184.

Gold, J.I. \& Shadlen, M.N. (2000) Representation of a perceptual decision in developing oculomotor commands. Nature, 404, 390-394. 
Goldman-Rakic, P.S. (1996) Regional and cellular fractionation of working memory. Proc. Natl Acad. Sci. USA, 93, 13473-13480.

Grammont, F. \& Riehle, A. (2003) Spike synchronization and firing rate in a population of motor cortical neurons in relation to movement direction and reaction time. Biol. Cybern., 88, 360-373.

Hanes, D.P. \& Schall, J.D. (1996) Neural control of voluntary movement initiation. Science, 274, 427-430.

Ivry, R.B. (1996) The representation of temporal information in perception and motor control. Curr. Opin. Neurobiol., 6, 851-857.

Janssen, P. \& Shadlen, M.N. (2005) A representation of the hazard rate of elapsed time in macaque area LIP. Nat. Neurosci., 8, 234-241.

Kubota, K. \& Hamada, I. (1979) Preparatory activity of monkey pyramidal tract neurons related to quick movement onset during visual tracking performance. Brain Res., 168, 435-439.

Lecas, J.C., Requin, J., Anger, C. \& Vitton, N. (1986) Changes in neuronal activity in the monkey precentral cortex during preparation for movement. J. Neurophysiol., 56, 1680-1702.

Leon, M.I. \& Shadlen, M.N. (2003) Representation of time by neurons in the posterior parietal cortex of the macaque. Neuron, 38, 317-327.

Lucchetti, C. \& Bon, L. (2001) Time-modulated neuronal activity in the premotor cortex of macaque monkeys. Exp. Brain Res., 141, 254260.

Mangels, J.A., Ivry, R.B. \& Shimizu, N. (1998) Dissociable contributions of the prefrontal and neocerebellar cortex to time perception. Brain Res. Cogn. Brain Res., 7, 15-39.

Mauk, M.D. \& Buonomano, D.V. (2004) The neural basis of temporal processing. Annu. Rev. Neurosci., 27, 307-340.

Mauritz, K.H. \& Wise, S.P. (1986) Premotor cortex of the rhesus monkey: neuronal activity in anticipation of predictable environmental events. Exp. Brain Res., 61, 229-244.

Mazurek, M.E., Roitman, J.D., Ditterich, J. \& Shadlen, M.N. (2003) A role for neural integrators in perceptual decision making. Cereb. Cortex, 13, 12571269.

Nawrot, M., Aertsen, A. \& Rotter, S. (1999) Single trial estimation of neuronal firing rates from single-neuron spike trains to population activity. J. Neurosci. Meth., 94, 81-92.

Niki, H. \& Watanabe, M. (1979) Prefrontal and cingulate unit activity during timing behavior in the monkey. Brain Res., 171, 213-224.

Renoult, L., Roux, S. \& Riehle, A. (2004) Time is a rubberband: neuronal activity in monkey motor cortex in relation to time estimation. Soc. Neurosci. Abstr., 30, 550.2.
Requin, J., Brener, J. \& Ring, C. (1991) Preparation for action. In Jennings, R.R. \& Coles, M.G.H. (Eds), Handbook of Cognitive Psychophysiology: Central and Autonomous Nervous System Approaches. Wiley \& Sons, New York, pp. 357-448.

Riehle, A. (2005) Preparation for action: one of the key functions of the motor cortex. In Riehle, A. \& Vaadia, E. (Eds), Motor Cortex in Voluntary Movements: a Distributed System for Distributed Functions. CRC-Press, Boca Raton, FL, pp. 213-240.

Riehle, A., Grammont, F., Diesmann, M. \& Grün, S. (2000) Dynamical changes and temporal precision of synchronized spiking activity in monkey motor cortex during movement preparation. J. Physiol. (Paris), 94, 569-582.

Riehle, A., Grün, S., Diesmann, M. \& Aertsen, A. (1997) Spike synchronization and rate modulation differentially involved in motor cortical function. Science, 278, 1950-1953.

Riehle, A. \& Requin, J. (1993) The predictive value for performance speed of preparatory changes in activity of the monkey motor and premotor cortex. Behav. Brain Res., 53, 35-49.

Roux, S., Coulmance, M. \& Riehle, A. (2003) Context-related representation of timing processes in monkey motor cortex. Eur. J. Neurosci., 18, 1011-1016.

Schmidt, E., Gilbert Jost, R. \& Davis, K.K. (1974) Cortical cell discharge patterns in anticipation of a trained movement. Brain Res., 75, 309-311.

Shadlen, M.N. \& Newsome, W.T. (2001) Neural basis of a perceptual decision in the parietal cortex (area LIP) of the rhesus monkey. J. Neurophysiol., 86, 1916-1936.

Simon, S.R., Meunier, M., Piettre, L., Berardi, A.M., Segebarth, C.M. \& Boussaoud, D. (2002) Spatial attention and memory versus motor preparation: premotor cortex involvement as revealed by fMRI. J. Neurophysiol., 88, 2047-2057.

Staddon, J.E. \& Higa, J.J. (1999) Time and memory: towards a pacemaker-free theory of interval timing. J. Exp. Anal. Behav., 71, 215-251.

Tanji, J. \& Evarts, E.V. (1976) Anticipatory activity of motor cortex neurons in relation to direction of an intended movement. J. Neurophysiol., 39, 10621068.

Thach, W.T. (1978) Correlation of neural discharge with pattern and force of muscular activity, joint position, and direction of intended next movement in motor cortex and cerebellum. J. Neurophysiol., 41, 654-676.

Vaadia, E., Kurata, K. \& Wise, S.P. (1988) Neuronal activity preceding directional and nondirectional cues in the premotor cortex of rhesus monkeys. Somatosens. Mot. Res., 6, 207-230.

Wang, X.J. (2001) Synaptic reverberation underlying mnemonic persistent activity. Trends Neurosci., 24, 455-463. 